Apidologie, 1977, 8 (1) 33-59.

\title{
L'ESSAIMAGE CHEZ LES HYPOTRIGONES AU GABON DYNAMIQUE DE QUELQUES POPULATIONS
}

\author{
Das Schwärmen bei den Hypotrigonen in Gabun \\ Die Dynamik einiger Populationen
}

Roger DARCHEN

Station biologique, 24620 les Eyzies, France

\begin{abstract}
SUMMARY
SWARMING OF AFRICAN STINGLESS BEES (Hypotrigona), LAYING OF THE QUEEN AND DYNAMIC OF SOME YOUNG COLONIES
\end{abstract}

The observations have been made in Gabon between 1963 and 1975.

Thanks to the knowledge of some typical parameters of this area such as temperature, rainfall, air humidity, flower cycles, we can explain how there is no swarming during some seasons.

Stingless bees swarming goes through four stages during which we can observe a more or less sudden variation between the respective numbers of workers belonging to the three divisions of age that we have clearly defined at the beginning of the work. From the beginning old honey-gathering workers come into the new nest and initiate succinct fittings of the habitation. Then, the same workers with some younger ones begin to set up storing cells. Later we can see that the number of young workers increases while the number of the elders reduces in correlation. These ones go on fitting up the nest. Finally the last period begins when a virgin queen comes with many young workers. At this moment they start to build brood cells. The queen is fecundated and quickly lays eggs. The stereotype laying behaviour of the queen has been observed in details.

As soon as the young colony is settled up, we notice that its weight grows steadily. But during this time the weight of old hives reduces. This loss of weight of old hives occurs during all the swarming period and goes on during the following months which, furthermore are correlative to the global reduction of flowering.

All these notes allow interesting comparisons between stingless bees and Apis mellifica. 


\section{RÉSUMÉ}

Les observations ont été effectuées au Gabon entre 1963 et 1975.

Grâce à la connaissance de divers paramètres caractéristiques de cette région, à savoir, température, pluies, humidité de l'air, cycles de floraisons, on peut expliquer pourquoi, à certaines saisons, il n'y a pas d'essaimage.

L'essaimage des Trigones se déroule en 4 étapes au courä desquelles on assiste à une variation plus ou moins brusque des nombres respectifs des ouvrières appartenant aux 3 classes d'âge définies au début du travail. Dès le début, on voit l'arrivée dans le nouveau nid de vieilles butineuses qui procèdent à l'aménagement sommaire de l'habitacle. Ensuite, les mêmes individus, accompagnés de quelques-uns, plus jeunes, commencent à édifier des cellules de réserve. Puis on assiste à une augmentation du nombre de jeunes ouvrières et corrélativement à une diminution du nombre des plus vieilles. Ces dernières continuent les travaux déjà commencés. Enfin l'ultime phase de l'essaimage débute par l'arrivée d'une reine vierge, accompagnée de très nombreuses jeunes ouvrières. Le travail de construction des cellules à couvain commence à ce moment là. La reine est fécondée et se met à pondre rapidement. Le comportement stéréotypé de ponte de la reine a pu être suivi en détail.

A partir du moment où la jeune colonie est installée, on constate que le poids de celle-ci croît régulièrement. Or, pendant le même temps, celui des ruches âgées diminue. Cette perte de poids des ruches âgées se manifeste durant toute la période d'essaimage et se prolonge pendant les mois qui suivent. Ces derniers correspondent d'ailleur.' à une diminution globale de la floraison.

Toutes ces observations permettent des comparaisons intéressantes entre les Trigones et Apis mellifica.

\section{I. - HISTORIQUE ET OBJECTIF DE CE TRAVAIL}

Vers le milieu du $19^{\mathrm{e}}$ siècle (1868), les entomologistes, en la personne de Peckolt, découvrirent que les colonies de Mélipones pouvaient essaimer à l'instar de l'abeille européenne, Apis mellifica. Cependant, on était encore loin de connaitre les différentes étapes et les détails de la fondation des nouvelles colonies. Il fallut attendre les travaux de Nogueira-Neto (1947, 1948, 1954), de KERR (1951) pour que les caractères propres et essentiels de l'essaimage des Trigones et des Mélipones soient dessinés.

Récemment, Terada (1972) a consacré une monographie l'essaimage d'une Trigone brésilienne, Frieseomelitta varia, et Fonseca a présenté une petite revue générale sur le sujet au $25^{\mathrm{e}}$ Congrès d'Apiculture de Grenoble (1975), reprenant ainsi en grande partie, la synthèse proposée par MourE, Nogueira-Neto et Moure au $10^{\mathrm{e}}$ Congrès International d'Entomologie de Montréal (1958).

Ces derniers auteurs nous ont montré que 1) la reine vierge essaime avec une partie de la population lorsque le nouvel habitacle a été suffisamment préparé par les exploratrices (elles ont bouché les crevasses du nid, amené de la cire, du miel et du pollen de la colonie mère), 2) des mâles volent nombreux autour de la nouvelle colonie en attendant de féconder la reine, 3) les relations entre la colonie mère et la colonie fille peuvent durer plusieurs jours, sinon plusieurs semaines ou plusieurs mois. 
Terada, de son côté, a observé le comportement des abeilles de deux essaims une semaine après leur installation dans leur ruche et fourni des renseignements sur le développement de ces nouvelles ruches durant un mois.

Ce travail, ici présenté, sur les essaimages d'Hypotrigona correspond à 12 années d'observations au Gabon où j'ai au moins possédé un rucher d'une centaine de colonies. J'ai pu ainsi suivre des dizaines d'essaimages naturels dès l'instant où les éclaireuses commençaient à s'intéresser à leur nouvel habitacle jusqu'au moment où les colonies filles avaient atteint leur état adulte. Enfin, des séjours à différentes saisons de l'année m'ont permis de connaitre les périodes d'essaimages et la structure des populations au cours de l'année.

\section{II. - LIEU D'OBSERVATION}

Ce travail a été effectué à Makokou (Gabon) dans le cadre d'un laboratoire propre du C.N.R.S.

La connaissance exacte du biotope est ici nécessaire pour comprendre les phénomènes liés à l'essaimage de ce pays.

La région de l'Ogoué-Ivindo est avant tout forestière et se trouve à quelques kilomètres au nord de l'Équateur. A part quelques rares lambeaux de forêt primaire qui existent encore çà et là, nous nous trouvons en présence d'une forêt secondaire assez dense. Elle est découpée par un large fleuve, l'Ivindo, et de nombreuses rivières et marigots qui en font un énorme massif forestier très humide. Il y a un seul grand axe routier et de nombreuses pistes. Les villages se situent le long des routes.

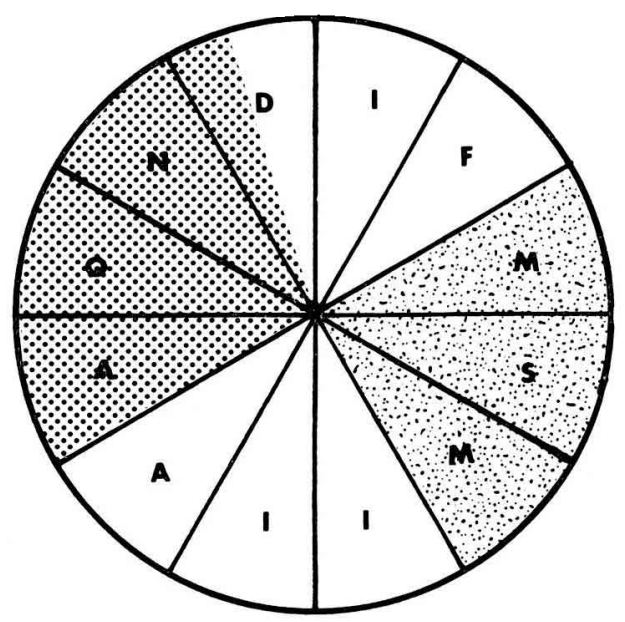

Fıg. 1. - Répartition des saisons au cours de l'année à Makokou (Gabon). Le plus gros pointillê régulier représente la grande saison des pluies, le plus petit pointillé irrégulier, la petite saison des pluies, le reste, les saisons sèches.

Авв. 1. - Die Jahreszeiten in Makokou (Gabun). Regelmässig grob punktiert = grosse Regenzeit. Unregelmässig schwach punktiert $=$ kurze Regenzeit. Der Rest $=$ Trockenperiode. 


\section{Le climat}

L'année est découpée par 2 saisons de pluie et par 2 saisons sèches, (fig. 1). L'une de ces dernières, appelée “ petite saison sèche 》, est en fait caractérisée par de longues périodes d'ensoleillement alternant avec quelques chutes d'eau. La grande saison sèche va de juin à août inclus; la grande saison des pluies commence en septembre et ne cesse que vers le 15 décembre; la petite saison sèche débute autour du 15 décembre, se poursuit jusqu'à la fin février, tandis que la petite saison humide dure trois mois à partir de mars.
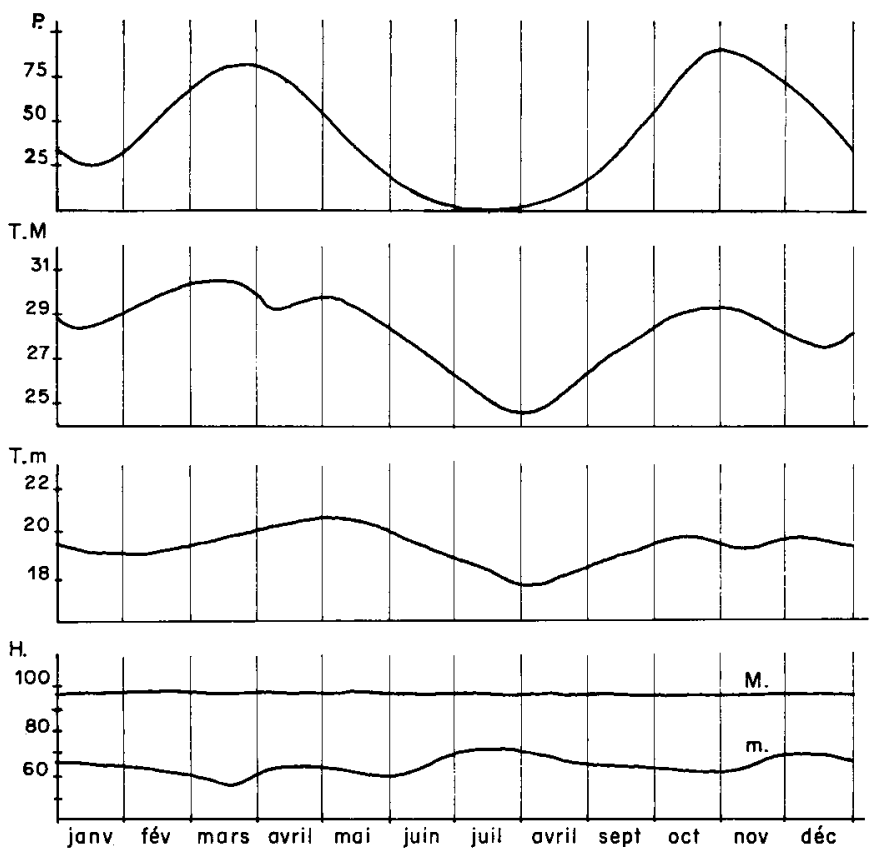

FIG. 2. - Courbes des variations mensuelles des chutes de pluie, de température et d'humidité durant 3 années (1962-1964) à Makokou. De haut en bas : pluies, températures maximales, températures minimales et humidité relative.

Aвв. 2. - Kurven der monatlichen Veränderungen von Regenfall, Temperatur und Feuchtigkeit während dreier Jahre (1962-1964) in Makokou. Von oben nach unten : Niederschläge, Höchsttemperaturen, Tiefsttemperaturen und rel. Feuchte.

Les courbes représentées ici - et établies par G. Dubost - sont des courbes moyennes de trois années 1962, 1963 et 1964 (fig. 2). Elles ont été enregistrées à l'aéroport de Makokou, c'est-à-dire dans un milieu largement dégagé, une sorte de clairière artificielle. On y découvre que les températures maxima ne dépassent pas $31^{\circ}$ durant la petite saison humide et que les minima n'excèdent pas $18^{\circ}$ durant la saison sèche. En revanche, l'atmosphère est 
souvent saturée et l'humidité relative est toujours au-dessus de $55 \%$. Il suffit de consulter les courbes précédentes et celle concernant l'insolation à Makokou établie par Charles-Dominique en 1968 (fig. 3).

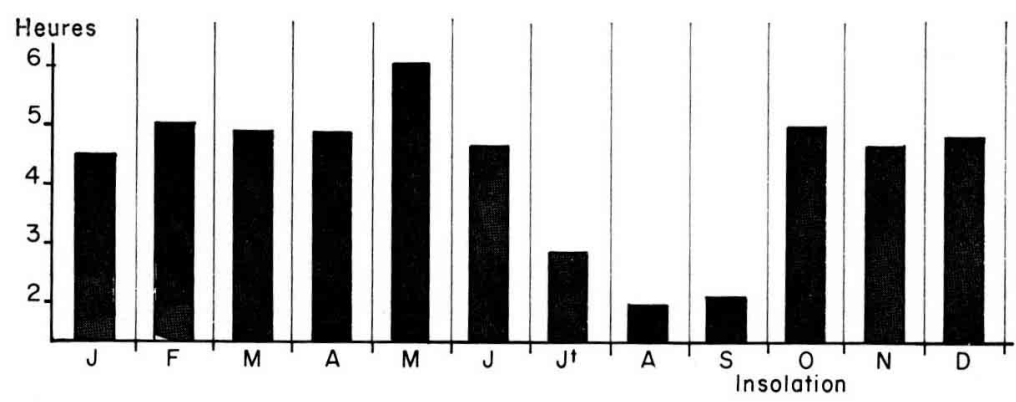

Fig. 3. - Moyennes mensuelles des heures d'insolation à Makokou en 1968.

Авв. 3. - Monatlicher Durchschnitt der Sonneneinstrahlung (Stunden) in Makokou (1968).

Notons que la courbe des moyennes d'humidité minimum ne varie pas parallèlement à celle des moyennes d'humidité maximum. Bien souvent les variations sont inversées. Le phénomène s'explique aisément si l'on sait que les saisons sèches correspondent à une insolation minimum et les saisons humides à une insolation maximum : l'évaporation est donc plus rapide et plus importante durant les saisons de pluies. Or, nous avons démontré, avec des araignées sociales, Agelena consociata, que ce fait est important car il semble conditionner la ponte des femelles à l'intérieur des nids : la ponte est presque inexistante durant les périodes d'humidité minimum.

Je n'ai, malheureusement, aucune courbe annuelle des moyennes des températures et d'humidité de la forêt dense à plus d'un mètre du sol. Cependant, les enregistrements que j'ai effectués pendant plusieurs mois presque au niveau du sol, donnent une idée des phénomènes météorologiques de ce milieu particulier où vivent les Hypotrigones. Les résultats donnés par divers enregistrements peuvent être résumés ainsi : les températures oscillent régulièrement entre $20^{\circ}$ et $25^{\circ}$ et l'humidité entre $80 \%$ et $95 \%$ (fig. 4).

Nous donnons ici la représentation de courbes typiques du milieu forestier.

\section{La floraison}

Pour compléter l'image de biotope où vivent les Hypotrigones de Makokou, il suffit d'ajouter la courbe de floraison et de fructification dessinée par CharLesDominique en 1968 à celles que nous possédons déjà (fig. 5). Cette courbe a été établie grâce à des relevés quotidiens de fleurs tombées sur une piste de 3 kilomètres à l'intérieur de la forêt d'Ipassa. On y découvre que les pics de floraison correspondent aux saisons de pluie : la diminution du nombre de 
fleurs récoltées en mars vient du fait qu'à cette période de cette année là, les chutes mensuelles de pluie ont été très réduites, $90 \mathrm{~mm}$ contre 80 en février et 225 en avril. Cette observation se révèlera capitale pour l'interprétation des résultats que nous allons présenter dans la suite de ce mémoire.
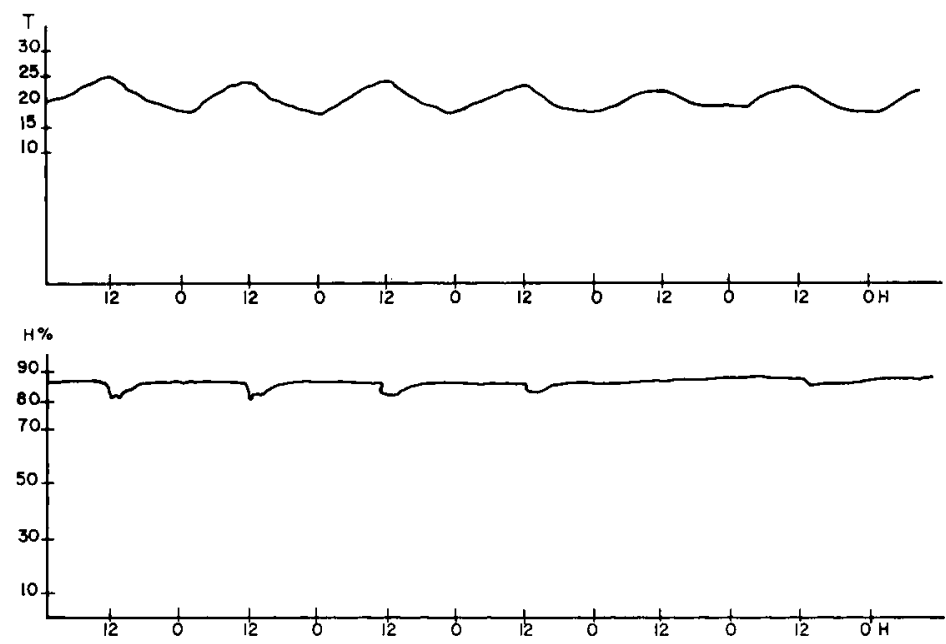

FIg. 4. - Courbes typiques de températures et d'humidité dans la forêt secondaire entre le sol et $100 \mathrm{~cm}$ à Makokou.

Авв. 4. - Typische Temperatur- und Feuchtigkeitskurven in den Sekundarwäldern zuischen Bodenoberfläche und $1 \mathrm{~m}$ Höhe; Makokou.

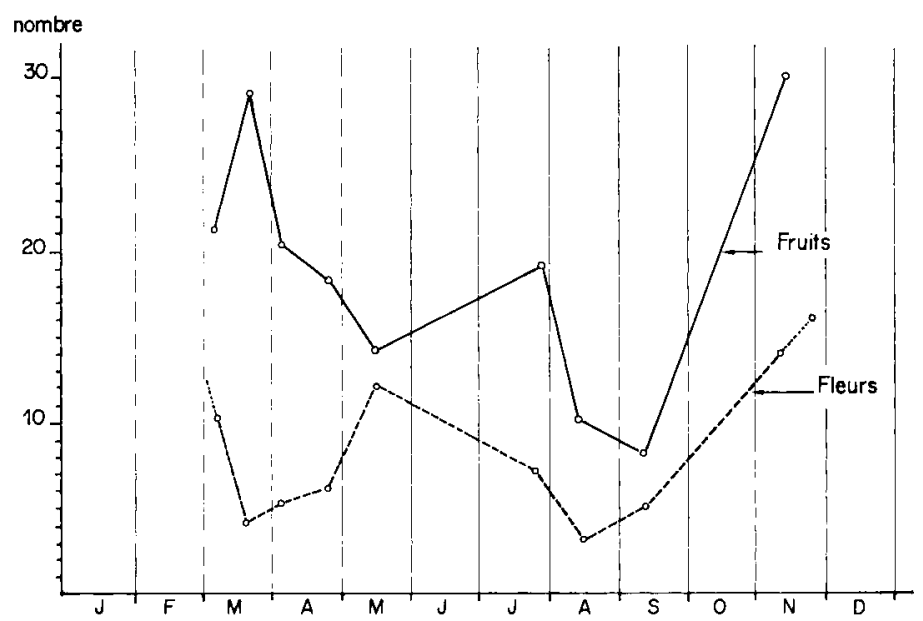

Fig. 5. - Variations annuelles de la floraison et de la fructification (données recueillies en 1968, d'après P. Charles-Dominique). Une communication personnelle de A. et M. Hladik permet d'inférer que la courbe continue son ascension de décembre (à droite) et commence à descendre en janvier (.- .-- - ).

Авв. 5. - Jährliche Veränderungen der Blüte- und Reifezeit. (Angaben aus 1968 nach P. Charles-Dominique.) Eine persönliche Mitteilung von A. und M. Hadik erlaubt die Annahme eines weiteren Ansteigens der Kurve im Dezember (rechts) und eines Absteigens im Januar. 


\section{Le biotope}

Les Hypotrigones vivent dans les branches creuses des arbres de la forêt. A cause de la densité du sous-bois formé d'arbrisseau, il est presque impossible de découvrir les nids de ces petites abeilles dont l'entrée se signale uniquement par un petit tube de cire de quelques millimètres de diamètre (fig. 6).

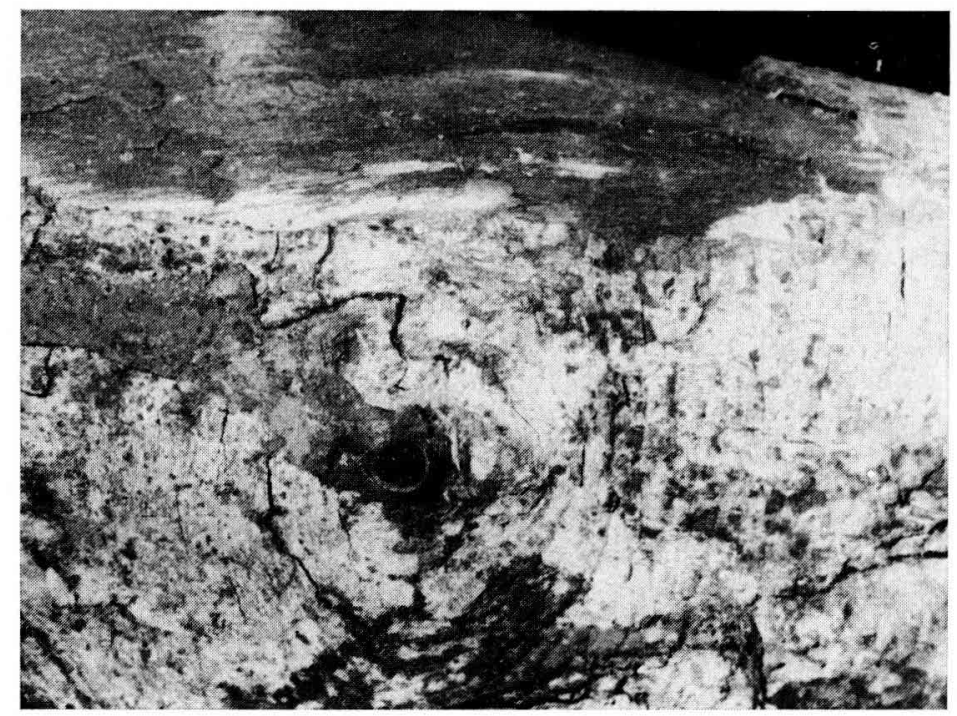

Fic. 6. - Tube d'entrêe d'un nid d'Hypotrigone dans le nœud d'un tronc d'arbre de la forêt.

Aвв. 6. - Eingangsröhre eines Hypotrigona - Nestes in der Astgabel eines Baumstumpfes im Wald.

En revanche, les cases d'habitation indigènes constituent une source intarissable de colonies. Les abeilles en effet se logent dans les poteaux de soutien, les chevrons en bambou et, surtout, les toits de feuillage des maisons. Malheureusement, depuis quatre ou cinq ans, les africains recouvrent leurs maisons de tôle ondulée et utilisent des chevrons des scieries à la place des bambous traditionnels. Il est donc, maintenant, devenu difficile de récolter aisément des nids d'Hypotrigones.

\section{La structure des nids}

On compte environ une demi-douzaine d'espèces d'Hypotrigones décrites jusqu'ici. Personnellement nous attendons de plus amples récoltes pour effectuer une révision de ce groupe dans lequel les espèces n'ont été distinguées entre elles que par des différences de tailles. Il faut reconnaître que c'est bien peu pour permettre une telle dichotomie parmi des insectes qui par ailleurs 
possèdent des nids dont la structure est très voisine, sinon similaire. Les nids ont été décrits à plusieurs reprises, mais c'est BASSINDALE qui nous en a donné la meilleure image.

Un nid d'Hypotrigone comprend 3 éléments architecturaux caractérisés : 1) le tunnel de circulation, 2) les cellules à couvain, 3) les amas de réserves.

1) Le nid s'ouvre par un tunnel de cire transparente de quelques millimètres à quelques centimètres de long et de quelques millimètres de diamètre. J'ai eu l'occasion d'expérimenter avec ce tube et découvrir que sa longueur et sa direction dépendent de l'éclairement extérieur. Plus le nid est dans l'ombre, plus les abeilles auront tendance à l'allonger vers la lumière; j'ai vu ainsi un tube d'une vingtaine de centimètres de long. Le diamètre non plus, n'est pas fixe : quelquefois le tube est cylindrique, quelquefois il a l'aspect d'une corne d'abondance. Ce tube d'envol se prolonge, à l'intérieur du nid, sur plusieurs centimètres par un autre tunnel plus large, fait de résine mêlée à de la cire. Ce tunnel intérieur est accolé à la paroi de la ruche (fig. 7). Un diaphragme de résine très dure percée de 2 à 4 orifices délimite la jonction des tubes extérieur et intérieur. Ces pertuis ne peuvent laisser passer qu'une seule abeille à la fois. Son rôle est certainement défensif. Les Hypotrigones ont, en effet, peu de moyens pour se protéger des parasites ou des prédateurs. Comme toutes les

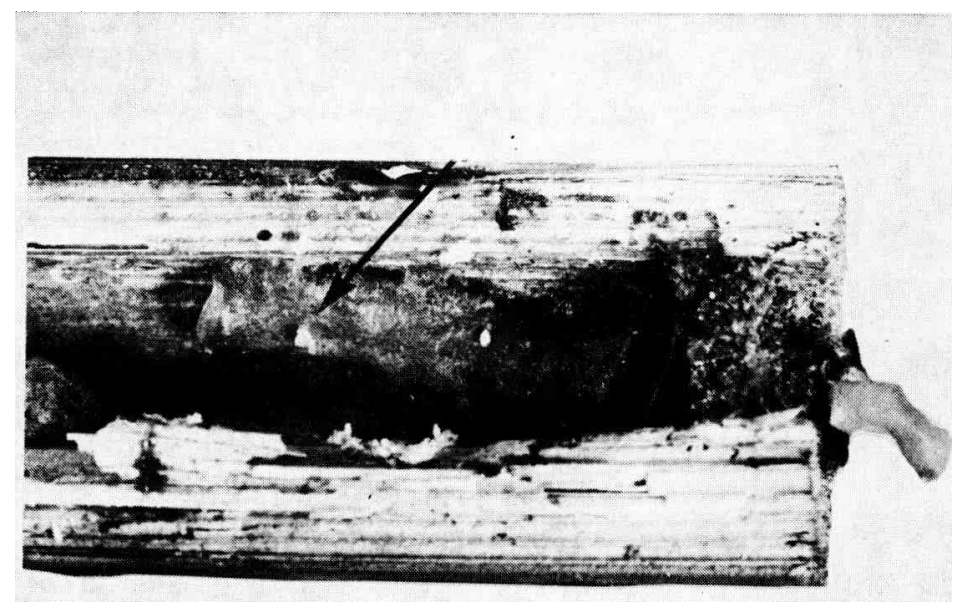

Fig. 7. - Détail du tunnel d'entrêe d'un nid d'Hypotrigone. On voit nettement le tube extérieur (à droite) transparent qui se prolonge à l'intérieur par un tunnel de couleur foncé collé à la paroi intérieure (flèche).

Aвв. 7. - Einzelheit des Eingangstunnels eines Hypotrigona - Nestes. Man sieht deutlich (rechts) die durchscheinende Röhre, de sich als dunkel gefärbter Tunnel ins Innere fortsetzt. Sie ist an die Innenwand angeklebt (Pfeil). 
Trigones, elles n'ont pas d'aiguillon, leur taille et leurs mandibules sont faibles. Elles ne peuvent que se précipiter sur les assaillants et les mordre ou bien boucher plus ou moins totalement l'orifice d'entrée de leur habitation.

2) A l'intérieur du nid, on trouve, répartis au hasard, des amas de cellules à couvain et des réserves alimentaires. Les cellules à couvain ont la forme de petits œufs, elles sont oblongues et de tailles identiques pour les ouvrières et les mâles. Les cellules de reines sont beaucoup plus grosses. Elles ne sont pas mêlées au couvain de mâles et d'ouvrières, mais disposées à la périphérie de l'amas de couvain. Les cellules à couvain sont attachées entre elles, vers le haut, vers le bas, latéralement ainsi qu'aux parois de la ruche grâce à des petits piliers de cire qui ne seront détruits qu'après la naissance des imagos lors de l'élimination totale des cocons. Ces derniers seront jetés à l'extérieur du nid. Observés à travers une ruchette vitrée, et sous un certain angle, les amas de cellules de couvain sont en général assez structurés, ils se présentent, en fait, comme des superpositions de couches de cellules relativement parallèles. Les distances entre les cellules sont régulières, elles résultent de la construction des petits piliers de cire dont nous venons de parler. Cette structure, en définitive ordonnée, de l'ensemble est compréhensible puisque les ouvrières constructrices édifient toujours des cellules de taille comparable dont les grands axes sont parallèles et à égale distance les unes des autres (fig. 8).

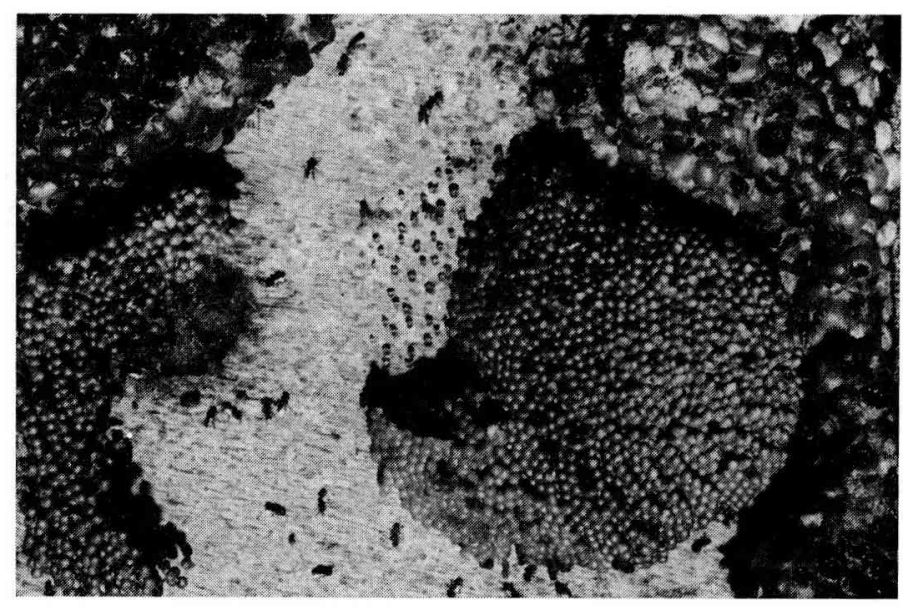

Frg. 8. - Structure d'un nid expêrimental d'Iypotrigone, vue au travers d'une paroi transparente (plexiglas). Le couvain en couches assez parallèles se trouve à gauche, les réservoirs à droite.

Aвв. 8. - Bau eines Hypotrigona - Versuchsnestes, durch eine transparente Plexiglaswand gesehen. Die in ziemlich parallelen Schichten (Lagen) angelegte Brut befindet sich links, die Futtervorräte rechts.

Chez les Hypotrigones, le couvain n'est pas entouré par des enveloppes de cire. En revanche, il est intéressant pour notre sujet d'étude de savoir que, 
très souvent, l'habitacle des abeilles est divisé en plusieurs compartiments au moyen de parois de résine surtout, de cire et même de débris de quartz comme je l'ai constaté avec étonnement à la loupe binoculaire. Nous comprendrons l'utilité de ce compartimentage lorsque nous entreprendrons la description des diverses étapes de l'essaimage. BAssindale signale en outre de petits tas de cire informes dans sa ruche expérimentale. Nous n'avons jamais remarqué cela dans les nids que nous avons ouverts ou dans les colonies que nous avons élevées.

3) Enfin les réserves de miel et de pollen sont disséminées au hasard, semble-t-il, dans des cellules entassées les unes sur les autres en différents endroits de l'habitacle. L'extraction du miel pur des ruches sauvages est donc très difficile parce qu'elle exige une filtration pour éliminer le pollen.

\section{III. - LE MATÉRIEL ET LES ÉLEVAGES}

Notre premier souci a été de constituer un important rucher abrité. Il a été possible, de la sorte, à tous instants, de contrôler les essaimages. Nous avons eu la chance de pouvoir transporter dans notre rucher un morceau de toit d'une case abandonnée qui contenait des dizaines de nids d'Hypotrigone. Plusieurs colonies ont été immédiatement introduites dans divers types de ruche vitrée dont il est inutile de donner des détails précis tant les goûts de ces abeilles sont éclectiques. Rappelons que Bassindale avait déposé sa colonie dans une assiette à soupe recouverte d'une plaque de verre! Nous avons installé nos insectes, soit dans de petites boîtes transparentes en plexiglas, soit dans des tubes de verre de $5 \mathrm{~cm}$ de diamètre et d'une trentaine de centimètres de long, soit enfin dans des boîtes formées d'un cadre de bois de 1 à $3 \mathrm{~cm}$ d'épaisseur fermé des deux côtés par des plaques transparentes de plexiglas. Dans tous les cas, évidemment, nous avions ménagé un trou de sortie pour les insectes. Toutes les ruchettes pleines ou vides étaient déposées sur des étagères disposées en rayonnage.

Les opérations de transfert des colonies étaient en général couronnées de succès si l'on prenait les précautions nécessaires pour éviter l'invasion des ruches par deux groupes de parasites, les fourmis Monomorium et, surtout, deux espèces de coléoptères Nitudilides.

\section{IV. - LA PÉRIOdE D'ESSAIMAge}

Il existe plusieurs manières de connaître les périodes d'essaimage, 1) la première demande la présence sur les lieux au moins pendant les 12 mois d'une même année (cela n'a jamais été mon cas) ou bien à des périodes différentes de l'année et sur plusieurs années (en 12 années, je n'ai jamais vécu au Gabon durant les mois d'août et septembre); 2) la deuxième exige l'examen de plusieurs colonies récoltées à différents mois : l'absence de cellules royales et de couvain de mâles sont alors le signe indubitable qu'aucun essaim ne se prépare. Connaissant la biologie de nos Apides sociaux européens, on doit pouvoir affirmer que la réciproque n'est pas obligatoirement vraie car la présence de mâles n'indique pas nécessairement un prochain essaimage, mais seulement la possibilité de fécondations lors de (" remérages ) accidentels. On peut enfin 3) tenir un compte rigoureux des ruches vides ou pleines à chaque départ 
d'Afrique et à chaque retour au laboratoire. Sachant que les Hypotrigones, comme la plupart des Trigones, essaiment le plus près possible de leur rucher d'origine, il est peu probable que les essaims se soient évadés en dehors du rucher couvert pendant l'absence de l'observateur.

En utilisant plusieurs de ces points de repères, il nous a été possible de déterminer avec assez de précision la période d'essaimage au cours de l'année. 1) En déposant régulièrement des ruchettes vides sur les étagères de notre rucher, nous avons vu qu'elles pouvaient être colonisées dès le milieu de novembre jusqu'au début de mai. Le maximum d'occupations ayant lieu en décembre et en janvier. Il y a des tentatives d'essaimages sans succès au milieu de mai et au début de juin. 2) Nous n'avons malheureusement que peu d'analyses de populations déjà bien établies dans leur ruche depuis une année; toutefois nous avons remarqué que les mois d'essaimage correspondent à la présence de mâles dans les ruches. Ainsi nous avons trouvé respectivement $2 \%$ de mâles en janvier, $8 \%$ en février, $1 \%$ en avril, $0,5 \%$ en juin, $0 \%$ en juillet. En revanche, aucun essaim de l'année ne contenait des mâles avant ou après la fécondation des reines.

Le phénomène de l'essaimage est donc à cheval sur la fin de la grande saison des pluies, la petite saison sèche et la petite saison des pluies. La lecture des courbes (fig. 3,4 et 5 ) nous montre qu'il coïncide avec les maxima de température, d'humidité relative, de pluviosité et d'insolation. On voit en outre que l'absence d'essaimage se situe durant la grande saison sèche, pendant laquelle les moyennes de température minimum descendent au-dessous de $19^{\circ}$, celles de l'humidité à moins de $60 \%$ et l'insolation se réduit à 2 heures en tout par jour. Ces résultats ne nous surprennent pas, car nous avons constaté que l'activité des abeilles à ce moment là est très réduite; leurs sorties sont plus rares. Fait intéressant encore et visible sur la courbe de floraison, le nombre de fleurs diminue et certainement, d'après nos connaissances de botanique européenne, leur valeur nutritive également. D'ailleurs le tableau 4 nous montre la diminution graduelle des réserves des colonies bien établies dans leur ruche au fur et à mesure de l'approche de la grande saison sèche. Notons que les Gabonais de l'Ogoué-Ivindo recueillent du miel d'Apis mellifica adansonii au mois de janvier lorsque leur maïs est en fleur et que les réserves sont aussi au maximum. Il est très possible que les rythmes biologiques d'Apis coïncident avec ceux des Hypotrigones que nous étudions ici.

\section{V. - ANALYSE DE L'ESSAIMAGE}

Comme on l'a vu précédemment, les essaimages des Trigones ne s'effectuent pas de la même manière que ceux de l'abeille domestique, Apis mellifica : ils sont progressifs. Or, c'est ce phénomène que nous avons tenté d'analyser avec plus de précision que nos prédécesseurs. 


\section{a. - L'âge des ouvrières}

Pour connaître l'âge des abeilles, le travail est facilité par le fait que l'abdomen de ces insectes n'acquiert pas immédiatement sa couleur définitive : ambre clair au début, il devient noir à la fin de son existence. De plus, la longueur de l'abdomen diminue en fonction de l'âge : il dépasse l'extrémité des ailes à la naissance de l'insecte, il est nettement caché sous elles à la fin de la vie de l'animal. Bassindale a suffisamment décrit ce phénomène lors de son travail sur Trigona (Hypotrigona) gribodoi. Pour la commodité de notre analyse, nous avons arbitrairement classé les abeilles adultes en 3 groupes, les jaunes clair (ce sont les abeilles naissantes, et les nourrices), les marrons (les ouvrières qui s'occupent des réserves et du nettoyage du nid) et en fin les noires (les exploratrices, les butineuses). Nous n'avons pas parlé des cirières parce qu'elles sont à cheval sur les 2 premiers groupes. Ces 3 catégories que nous définissons correspondent au 5 classes établies par Bassindale selon le schème ci-joint (fig. 9).

\begin{tabular}{|c|c|c|c|c|}
\hline GROUPE & TRAVAIL & TERGITE ABDOMINALE & $\begin{array}{c}\text { AILE } \\
\text { ABDOMEN }\end{array}$ & GROUPE \\
\hline GRUPPE & ARBEIT & ABDOMINAL TERGIT & $\begin{array}{c}\text { FLUGEL } \\
\text { ABDOMEN } \\
\end{array}$ & GRUPPE \\
\hline 1 & $\begin{array}{l}\text { Aucun } \\
\text { Keine }\end{array}$ & & de profil & \multirow{2}{*}{$\begin{array}{c}J \\
\text { Gelb }\end{array}$} \\
\hline 2 & $\begin{array}{l}\text { Nourrices } \\
\text { Ammenbienen }\end{array}$ & & & \\
\hline 3 & $\begin{array}{l}\text { Magasinières } \\
\text { Baubienen }\end{array}$ & & & \multirow{2}{*}{$\begin{array}{c}\text { M } \\
\text { Braun }\end{array}$} \\
\hline 4 & $\begin{array}{l}\text { Nettoyeuses } \\
\text { Putzbienen }\end{array}$ & & & \\
\hline 5 & $\begin{array}{l}\text { Butineuses } \\
\text { Trachtbienen }\end{array}$ & m- & de profil & $\begin{array}{c}N \\
\text { Schwarz }\end{array}$ \\
\hline
\end{tabular}

FIg. 9. - La coloration et l'âge des ouvrières d'après Bassindale.

Aвв. 9. - Farbe und Alter der Arbeiterinnen nach Bassindale. 


\section{b. - Les étapes de l'essaimage}

On peut distinguer 4 étapes dans l'essaimage depuis l'arrivée des premières exploratrices jusqu'au moment où la reine commence à pondre ses premiers œufs. Nous les avons étudiées en recueillant de temps en temps tous les individus de la colonie et en observant les comportements.

\begin{tabular}{|c|c|c|c|}
\hline $\begin{array}{ll}\text { TAB. } & 1 \\
\text { neuer } & V\end{array}$ & $\begin{array}{l}\text { 1. } \\
\text { Völker in } \\
\end{array}$ & $\begin{array}{l}\text { yse der } \\
\text { schiedenen } \\
\text { ärmens. }\end{array}$ & $\begin{array}{l}\text { Populationen } \\
\text { Stadien des }\end{array}$ \\
\hline$=\therefore-=$ & $\bar{T}=\overline{=}$ & $=-\cdots=$ & $\overline{-}=--$ \\
\hline Stades & Jaunes & Marrons & Noires \\
\hline Stadien & Gelb & & Schwarz \\
\hline II & $\begin{array}{r}10 \\
233 \\
73 \\
42\end{array}$ & $\begin{array}{r}57 \\
0 \\
178 \\
14\end{array}$ & $\begin{array}{r}287 \\
268 \\
116 \\
22\end{array}$ \\
\hline III & $\begin{array}{r}380 \\
139 \\
616 \\
186 \\
78 \\
205 \\
139\end{array}$ & $\begin{array}{r}138 \\
37 \\
292 \\
77 \\
44 \\
87 \\
37\end{array}$ & $\begin{array}{r}99 \\
54 \\
77 \\
157 \\
27 \\
0 \\
54\end{array}$ \\
\hline IV & $\begin{array}{l}566 \\
206 \\
669 \\
443 \\
400 \\
382 \\
235 \\
830 \\
366 \\
739 \\
463 \\
452 \\
273 \\
186 \\
229 \\
789 \\
400 \\
233 \\
566 \\
382 \\
207 \\
767\end{array}$ & $\begin{array}{r}286 \\
74 \\
227 \\
27 \\
39 \\
116 \\
44 \\
241 \\
58 \\
241 \\
163 \\
187 \\
209 \\
77 \\
129 \\
108 \\
39 \\
117 \\
286 \\
116 \\
84 \\
140\end{array}$ & $\begin{array}{r}288 \\
0 \\
316 \\
19 \\
22 \\
192 \\
39 \\
127 \\
193 \\
308 \\
162 \\
287 \\
170 \\
157 \\
144 \\
299 \\
22 \\
99 \\
288 \\
192 \\
74 \\
161\end{array}$ \\
\hline 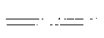 & $-=$ & $=$ & $\ldots$ \\
\hline
\end{tabular}

Le tableau 1 présente les résultats des analyses des populations recueillies dans les ruches au cours des essaimages. 


\section{Première étape}

En pleine période d'essaimage, des ruchettes, qui ont déjà servi, imprégnées de l'odeur de la cire, du miel peuvent être explorées dès les premières heures de leur installation dans le rucher et colonisées dès le lendemain. Les boîtes en plastique, couleur bleue foncée, sont généralement choisies de préférence à celles peintes en vert d'herbe. Très rapidement, pendant le jour des exploratrices deviennent des constructrices car elles se mettent à édifier le tube en cire d'entrée de la ruche, d'autres se contentent d'en garder l'orifice. Toutes ces abeilles ont l'abdomen noir. Ce sont donc les adultes les plus vieux de la colonie ( 5 et dernier stade de Bassindale). Vers la fin de la période d'essaimage, ces abeilles peuvent occuper la nouvelle habitation pendant plusieurs semaines; leur nombre fluctue généralement. Il arrive souvent, alors, que les colonisatrices ne passent pas à la seconde étape de l'essaimage et qu'elles abandonnent définitivement le nid.

L'occupation du nid est quelquefois rendue cifficile par le fait que sa structure est à modifier afin de l'isoler du monde extérieur. L'enceinte est souvent trop vaste pour les quelques dizaines d'abeilles qui vont l'habiter. Les exploratrices se mettent alors à en diminuer la capacité par la construction d'une cloison de résine et de cire. Il est très fréquent de rencontrer cet élément d'architecture dans les nids d'Hypotrigones. Lorsque la population devient plus dense la cloison est alors plus ou moins détruite afin de libérer un espace plus grand.

Mais le cas le plus exceptionnel que j'ai pu observer est celui de la colonisation d'une cloche en bois qui est normalement attachée au cou des chiens pendant la chasse. Laissée sur un mur, près du laboratoire, depuis quelques semaines, elle reçut la visite des exploratrices qui se mirent à l'aménager. Le gros problème pour elles fut d'obturer l'orifice de la cloche qui représentait une surface de $36,75 \mathrm{~cm}^{2}(3,5 \mathrm{~cm} \times 10,5 \mathrm{~cm})$. Les abeilles commencèrent à fixer tout autour de l'ouverture un mélange de résine, de cire, de fins cristaux de quartz et de fibres végétales, puis, dans toutes les directions centripètes et simultanément, des dizaines d'abeilles construisirent une paroi de cire plus pure, c'est-à-dire comprenant moins d'éléments étrangers que sur le pourtour. Un des faits les plus intéressants lors de cette construction fut, sans nul doute, l'aménagement progressif du tube de sortie au niveau de la membrane de cire bien avant sa fermeture définitive. Tandis que la paroi obturait déjà la moitié de l'ouverture, les abeilles interrompirent leur travail de construction au milieu de la partie droite, puis elles découpèrent lentement un lambeau de cire dans la paroi déjà construite. Le fragment d'opercule enlevé était situé à l'extrême droite, là où il prenait appui sur le bord de la cloche. A partir de l'ouverture ainsi aménagée, le tunnel d'entrée fut rapidement édifié. La tota- 
lité du travail dura à peine 3 jours. Au cours des jours suivants, la paroi de cire fut épaissie régulièrement (fig. 10).

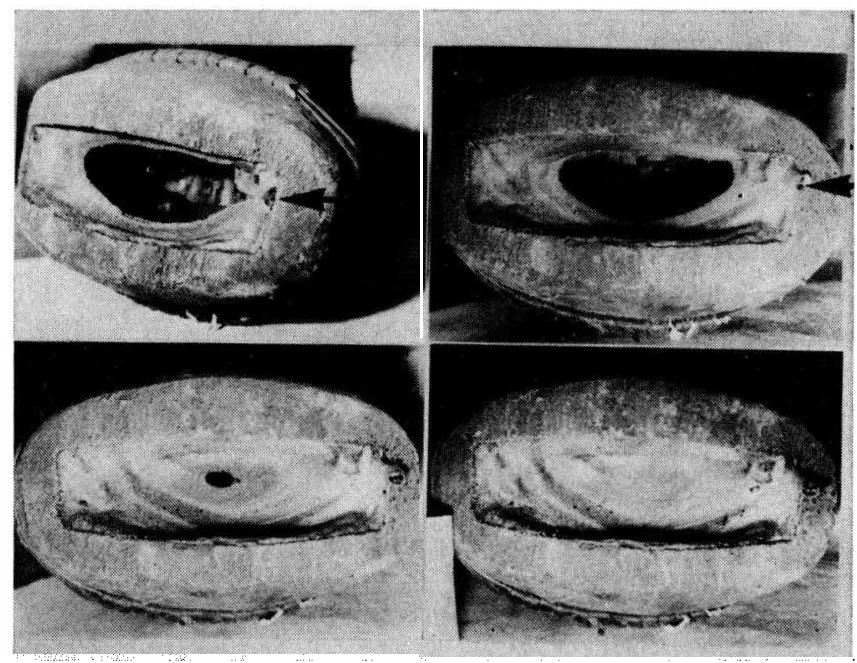

FIG. 10. - L'occupation d'une cloche on bois de chien de chasse. De gauche à droite et de haut en bas, les abeilles exploratrices édifient une paroi de cire, de résine et de quartz pour former une niche habitable. Remarquer sur la droite de l'ouverture de la cloche l'aménagement du futur tube de sortie dès les premières heures de la colonisation et des constructions (pointe de la flèche).

Авв. 10. - Die Besetzung einer hölzernen Jagdhund-Glocke. Von links nach rechts und von oben nach unten; die Kundschafterbienen errichten eine Wand aus Wachs, Harz und Quarz, um eine bewohnbare Nische zu schaffen. Man beachte rechts die Offnung der Glocke, die Anlage der künftigen Ausgangsröhre von der ersten Stunde der Besiedlung und des Baues an (Pfoilspitze).

\section{Deuxième étape}

C'est encore une période de préparation, mais ici un certain nombre d'abeilles, plus jeunes, arrivent dans la ruche. Il y a des individus des stades 2,3 et 4 de Bassindale. La proportion de vieilles abeilles est encore importante. Leur travail consiste à continuer les constructions précédentes (le tube, les membranes, le calfeutrage) et à commencer l'édification des cellules de réserve (miel surtout et pollen en quantité moindre). Le travail ici avance lentement, cependant il est nécessaire que cette étape soit de courte durée sinon l'essaimage définitif n'aura pas lieu. L'essaimage avorte d'autant plus facilement que l'on s'éloigne des mois de décembre et janvier. Cette deuxième étape, habituellement, ne dure que peu de jours.

\section{Troisième étape}

Brusquement, en l'espace de quelques heures, des dizaines de jeunes abeilles des stades 2 et 3 (Bassindale), envahissent la ruche alors que le 
nombre des ouvrières noires et marron foncé diminue. Le travail n'est pas très différent de celui de la $2^{\mathrm{e}}$ étape. Le nombre de cellules de réserves (miel et pollen) augmente un peu. Durant cette période une dizaine de cellules peuvent être construites. En fait, leur nombre est très variable suivant les colonies. Il est fonction de la quantité d'abeilles essaimantes. Le tableau 1 montre combien cette variabilité est grande. Le tunnel intérieur déjà décrit est édifié lentement à ce moment là.

\section{Quatrième étape}

Elle correspond à l'arrivée de la reine vierge entourée de centaines d'individus de tous les stades $2,3,4$ et 5 et peut-être 1 . Les abeilles les plus nombreuses sont les plus claires, c'est-à-dire les plus jeunes. Il est encore très difficile de distinguer la reine qui a presque la taille des jeunes ouvrières gonflées de miel et de pollen. La reine n'est généralement pas fécondée immédiatement. Les jeunes ouvrières en profitent pour commencer à construire rapidement les premières cellules à couvain, pendant que d'autres s'occupent des cellules de réserve qui augmentent très rapidement. Aux heures chaudes de la journée, les mâles des vieilles colonies volent par centaines autour de la ruche ou bien s'immobilisent en groupes sur des substrats voisins. La fécondation de la reine a lieu en général dans les 48 heures qui suivent son arrivée dans sa nouvelle habitation. L'activité constructrice de la ruche est alors décuplée : très rapidement on n'arrive plus à compter les cellules de couvain ou de réserve. Les ouvrières finissent alors $d$ 'isoler leur nid $d u$ monde extérieur plus hermétiquement en construisant à l'intérieur du tunnel d'entrée cette paroi de résine percée d'orifices plus petits que la taille de la reine. Le tube intérieur est prolongé de plusieurs centimètres. Dans ce conduit et son prolongement extérieur, les ventileuses se tiennent à la queue leu leu, l'extrémité de l'abdomen tourné vers la sortie, et ventilant activement lorsque la température intérieure de leur niche devient trop élevée.

\section{VI. - LA PONTE DE LA REINE D'HYPotRigona (fig. 11)}

\section{1. -- La construction des cellules à couvain}

Pendant que la reine se repose sur les réserves ou sur les cellules à couvain de son nid, plusieurs jeunes ouvrières sécrétant de la cire au niveau de leurs tergites abdominaux, construisent simultanément plusieurs cellules. Au repos, la reine est généralement entourée d'une cour de 3 à 15 ouvrières qui se tiennent à environ 5 à $10 \mathrm{~mm}$ d'elle. La reine se déplace de temps en temps à l'intérieur de ce cercle d'abeilles. Ces dernières se reculent rapidement à son approche. Des échanges trophallactiques ont lieu de temps en temps. Ils sont 
beaucoup plus fréquents que lorsque la reine marche tranquillement dans la ruche.

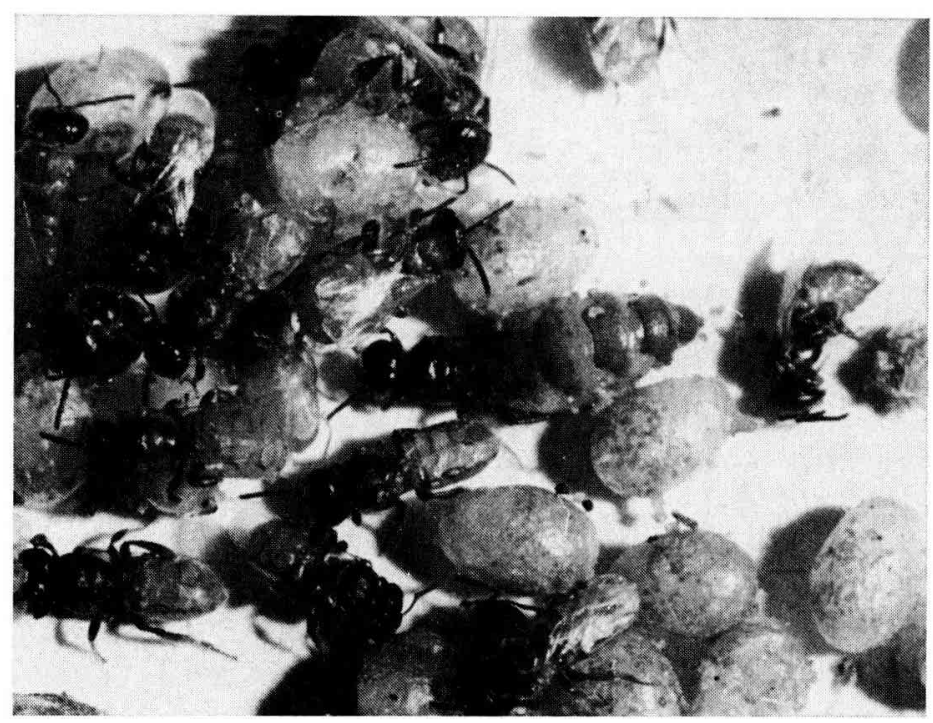

Fig. 11. - La reine et les ouvrières d'Hypotrigona.

Aвв. 11. - Königin und Arbeiterinnen von Hypotrigona.

\section{2. - La fréquence de la ponte de la reine}

Dans nos conditions expérimentales (en ruches vitrées) et durant la durée de l'observation (juin et juillet 1968, saison sèche), nous avons compté environ 2 ou 3 séquences de ponte par 24 heures. Le nombre d'œufs pondus à chaque séquence était variable et dépendait de la quantité de cellules construites par les ouvrières pendant les phases de repos de la reine. Il est donc possible qu'en période plus favorable la fréquence des séries de ponte n'augmente pas, mais que le nombre de cellules construites soit beaucoup plus grand. Nous savons qu'en Côte d'Ivoire certaines reines d'Hypotrigone peuvent pondre jusqu'à 82 œufs par 24 heures, dans les meilleures conditions expérimentales (tabl. 2).

\section{3. - La ponte de la reine}

Brusquement, la reine rompt son inactivité pour partir dans tous les sens et spécialement en direction du couvain. Elle visite les cellules en construction. Elle retrouve son repos si les constructrices n'ont pas encore suffisamment édifié de cellules. Elle peut recommencer ce manège plusieurs fois. Dans un des cas observés, il s'est écoulé environ 2 heures entre le premier déplacement et la première ponte. Lors de ses visites des cellules à couvain en construction, 
TABL. 2. - Exemple du temps passé par une reine lors de sa ponte dans plusieurs cellules construites simultanément et du nombre de " régurgitations » des ouvrières dans chaque cellule.

TAB. 2. - Beispiel der von einer Königin während der Eiablage in verschiedenen gleichzeitig gebauten Zellen verbrachten Zeit und der Anzahl der in jede Zelle Futter abgebenden Arbeiterinnen.

\begin{tabular}{|c|c|c|}
\hline Cellule No & $\begin{array}{c}\text { Durée } \\
\text { entre les pontes }\end{array}$ & $\begin{array}{c}\text { Nombre de } \\
\text { régurgi- } \\
\text { tations }\end{array}$ \\
\hline Zelle Nr. & $\begin{array}{l}\text { Zeit zwischen } \\
\text { d. Eiablage }\end{array}$ & $\begin{array}{c}\text { Zahl d. } \\
\text { Futterabgaben }\end{array}$ \\
\hline 1 & $5 \min 30 \mathrm{~s}$ & $?$ \\
\hline 2 & $4 \min$ & $?$ \\
\hline 3 & $3 \min$ & ? \\
\hline 4 & $15 \mathrm{~s}$ & $?$ \\
\hline 5 & $1 \min 50 \mathrm{~s}$ & 14 \\
\hline 6 & $3 \min 10 \mathrm{~s}$ & 15 \\
\hline 7 & $4 \min 30 \mathrm{~s}$ & 8 \\
\hline 8 & $5 \min 30 \mathrm{~s}$ & 19 \\
\hline 9 & $7 \min 30 \mathrm{~s}$ & 22 \\
\hline 10 & $9 \min 20 \mathrm{~s}$ & 15 \\
\hline 11 & $11 \min 10 \mathrm{~s}$ & 6 \\
\hline 12 & $13 \mathrm{~min} 30 \mathrm{~s}$ & 16 \\
\hline
\end{tabular}

la reine se fixe la tête en haut sur le bord de la cellule mais ne bat pas ses ailes si la cellule est inachevée. Elle repart en visiter d'autres en remuant ses ailes de temps en temps. Les ouvrières font de même. En revanche, lorsque les cellules ont acquis leur forme de poire, la reine se dandine rapidement, bat ses ailes très souvent, bouscule les ouvrières qui se trouvent sur son passage ou celles au travail au-dessus des cellules vides. Puis elle se fixe sur l'une des cellules après avoir tourné plusieurs fois autour de l'orifice. Ses antennes entrent souvent en contact avec celles des ouvrières au travail. Une cour de quelques individus se tient autour de la reine à ce moment là. Toujours fixée au bord de la cellule, elle continue à battre des ailes très fréquemment tandis que les nourrices vident leur estomac rempli d'un mélange de miel et de pollen, la fréquence des battements d'ailes de la reine diminue, semble-t-il, si le rythme de régurgitations des ouvrières est suffisamment rapide elle s'accélère si le travail des ouvrières est trop long. La reine effectue de nombreux échanges antennaires avec les ouvrières qui régurgitent le contenu de leur estomac. Assez souvent, elle vérifie le contenu des cellules et quelquefois elle s'y arrête longtemps comme si elle s'en nourrissait partiellement. La reine peut s'éloigner quelques instants de la cellule en cours de remplissage, toutefois elle se maintient sur le bord de cette cellule durant la plus grande partie du temps de régurgitation des aliments. Lorsque le liquide nourricier a atteint le niveau requis, la 
reine chasse les ouvrières et pond dans la cellule en une seconde environ. Elle va ainsi inspecter chaque cellule nouvellement construite et s'y fixer à son tour.

Le temps de remplissage des cellules, le nombre d'ouvrières qui se penchent sur les cellules, la durée entre 2 pontes, sont très variables. Prenons un exemple tableau 2. Il ressort de ce tableau que le temps écoulé entre les différentes pontes s'inscrit sur une courbe en $\mathrm{V}$ qui doit être la manifestation de l'intensité de l'excitation de la reine et des ouvrières au cours de la ponte. Elle part d'un minimum, passe par un maximum et, lentement retourne à un minimum beaucoup plus bas. En revanche, le nombre de régurgitations semble très variable. On peut supposer que la quantité de nourriture régurgitée par chaque abeille est inégale : le nombre de régurgitations doit donc différer en plus ou en moins en fonction de l'importance des dépôts individuels. Une observation attentive du comportement des individus a permis de découvrir en outre, que, à chaque remplissage d'une cellule, plusieurs abeilles exécutent des simulacres de régurgitations. Avec un peu d'expérience, il est facile de distinguer les simulacres des régurgitations véritables. En effet, lors d'un simulacre, les abeilles introduisent seulement la tête et une partie du thorax dans la cellule alors que pour une régurgitation vraie, les ouvrières y disparaissent presque totalement. Enfin, l'abdomen des premières ne se contracte pas tandis que celui des autres s'aplatit à la manière d'une poire sur laquelle on presserait. Lorsque le niveau du liquide nourricier atteint la hauteur nécessaire à l'intérieur de la cellule, la reine y dépose son ceuf. On n'a jamais observé, sur les Hypotrigones d'Afrique, d'oophagie nutricielle ni de la part de la reine, ni de celle des ouvrières.

\section{4. - L'operculation des cellules}

Aussitôt après la ponte, une seule des ouvrières parmi celles qui se trouvent juchées sur la cellule se met en devoir de la fermer. A cheval sur l'orifice, elle rabat, circulairement vers l'intérieur, une partie de la cire de la paroi de telle sorte que la cellule diminue de hauteur. Le sommet de la cellule s'arrondit, l'ouverture diminue de diamètre, et bientôt la coupole est totale, la cellule est définitivement close.

\section{5. - Le sens des constructions}

Dans les ruches en plein développement, les cellules sont construites dans toutes les directions possibles, mais les grands axes sont toujours verticaux. A cause de l'écartement assez standard des piliers séparant les cellules il apparaît en définitive des couches de cellules parallèles les unes aux autres. Ceci est particulièrement net dans un milieu isotrope comme l'est celui de nos ruches plates vitrées (fig. 8). 


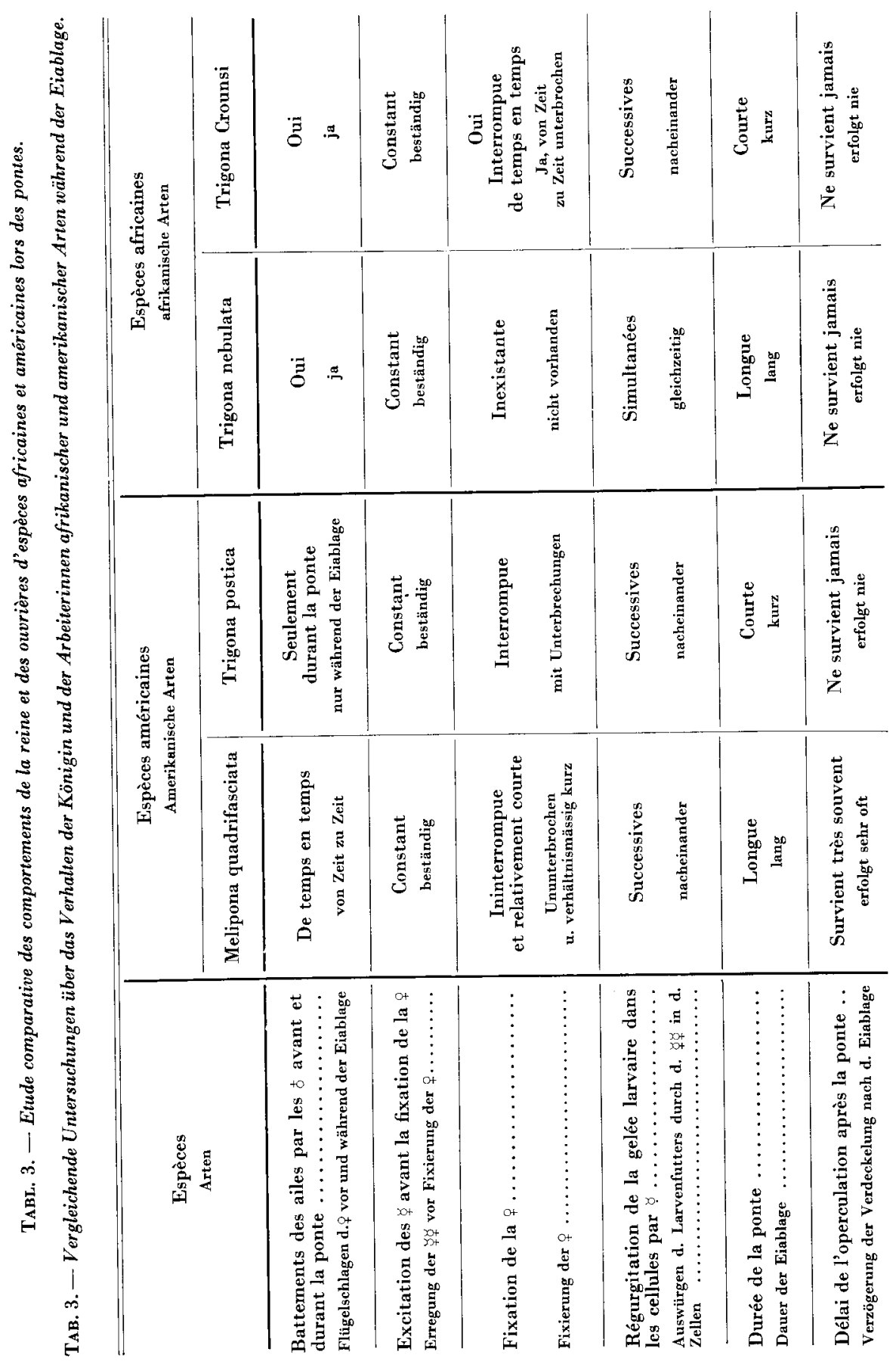




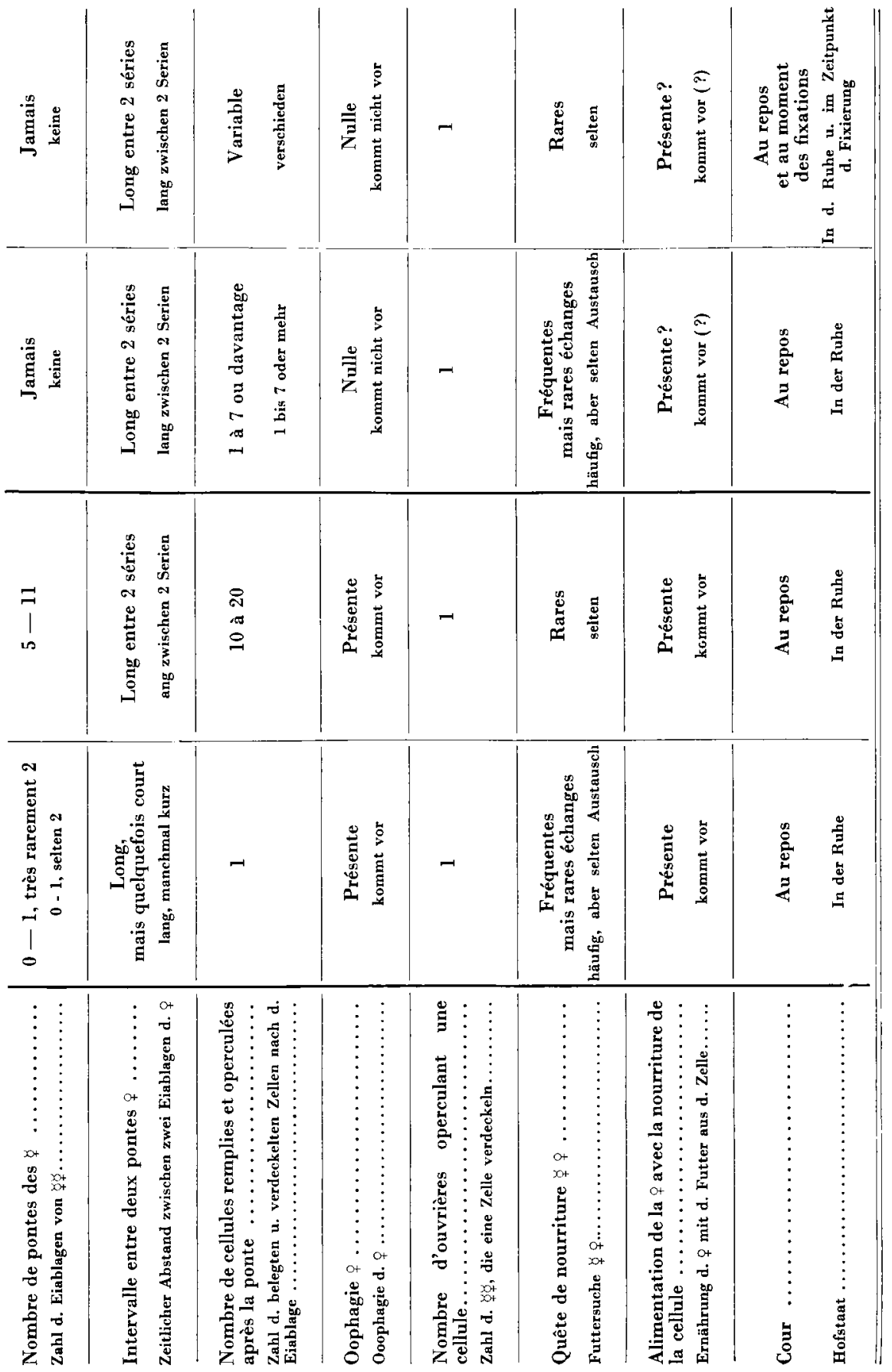


Le tableau 3, composé avec les résultats de nos propres travaux et ceux de SaKagami, montre les différences qui peuvent exister entre diverses espèces d'abeilles.

\section{VII. - LE DÉVELOPPEMENT ULTÉRIEUR DE LA JEUNE COLONIE}

A Makokou, dès 1968, nous avons pesé régulièrement, pendant 4 mois environ, des colonies installées dans leur ruche depuis l'année précédente et des colonies formées par des essaims récents.

Les résultats ont été collationnés dans les deux tableaux 4 et 5.

TABL. 4. - Variation de poids de vieilles colonies d'Hypotrigones sp.

ТАв. 4. - Gewichtsunterschiede bei alten Hypotrigona - Völkern.

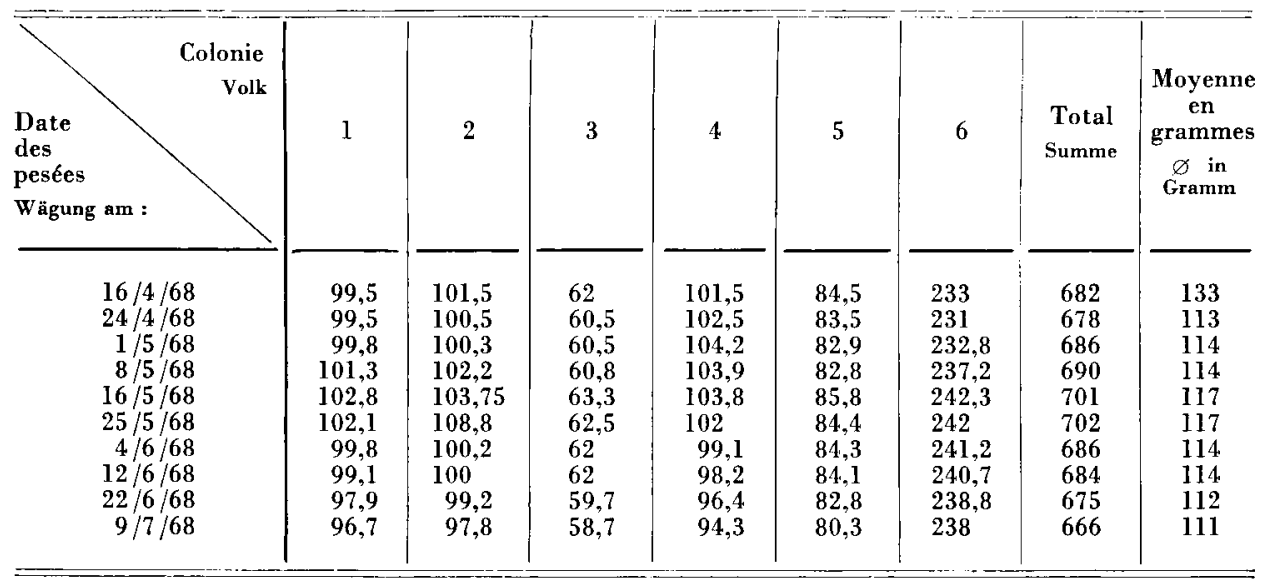

L'analyse des tableaux laisse apparaître les phénomènes très intéressants suivants, chez les vieilles colonies.

$1^{0}$ Après avoir augmenté régulièrement durant deux mois (petite saison des pluies) le poids de chaque colonie décroît jusqu'à la fin des expériences (saison sèche). Les résultats sont comparables à ceux de la Côte d'Ivoire.

$2^{\circ}$ La diminution du poids durant la saison sèche est lente. Évidemment des expériences seraient nécessaires pour comprendre ce phénomène différent. On peut toutefois en imaginer les causes : dans la région où sont faites ces observations, la température descend notablement durant la nuit; le jour, le ciel est gris, donc très peu d'ensoleillement. En saison sèche, il règne donc un froid relatif amenant chez les abeilles une sorte d'hibernation. Elles élèvent moins de couvain, butinent moins mais consomment moins aussi, les colonies 
menant véritablement une vie ralentie à ce moment-là, la chute des réserves est de ce fait moins spectaculaire.

TABL. 5. - Variation de poids de jeunes colonies d'Hypotrigones sp.

TAB. 5. - Gewichtsunterschiede bei jungen Hypotrigona - Völkern.

\begin{tabular}{|c|c|c|c|c|c|c|}
\hline $\begin{array}{l}\text { Colonie } \\
\text { Dolk } \\
\text { des } \\
\text { pesées } \\
\text { Wägung am }\end{array}$ & 1 & 2 & 3 & 4 & $\begin{array}{l}\text { Total } \\
\text { Summe }\end{array}$ & $\begin{array}{c}\text { Moyenne } \\
\text { en } \\
\text { grammes } \\
\varnothing \\
\text { in Gramm }\end{array}$ \\
\hline $\begin{array}{r}1 / 5 / 68 \\
8 / 5 / 68 \\
16 / 5 / 68 \\
25 / 5 / 68 \\
4 / 6 / 68 \\
12 / 6 / 68 \\
22 / 6 / 68 \\
9 / 7 / 68\end{array}$ & $\begin{array}{l}39 \\
41,8 \\
44,9 \\
45,8 \\
46,8 \\
47,3 \\
47,6 \\
48\end{array}$ & $\begin{array}{l}30,7 \\
32 \\
35 \\
36,8 \\
39,8 \\
42,1 \\
44,1 \\
45,1\end{array}$ & $\begin{array}{l}29 \\
29,5 \\
29,5 \\
29,5 \\
29,5 \\
29,9 \\
31\end{array}$ & $\begin{array}{l}36,5 \\
38,3 \\
39 \\
40,2 \\
40,6 \\
41,7 \\
42\end{array}$ & $\begin{array}{l}139 \\
148 \\
148 \\
156 \\
160 \\
163 \\
166\end{array}$ & $\begin{array}{l}35 \\
34 \\
37 \\
37 \\
39 \\
40 \\
40 \\
41\end{array}$ \\
\hline
\end{tabular}

Il est en revanche étonnant de constater que pendant la même période de l'année le poids des nouvelles colonies augmente régulièrement. Ce phénomène rappelle le comportement des nouveaux essaims de nos abeilles domestiques (Apis mellifica) qui en quelques jours construisent plusieurs rayons de cire, amassent du pollen et du miel dans des proportions in finiment plus grandes que celles des colonies établies dans leurs ruches depuis plusieurs mois. Chez les Trigones, nous avons vu que les colonies filles restent en général en relation constante avec la colonie mère; il leur est loisible d'emprunter des réserves à celle-ci pour constituer les leurs. Ici le phénomène est plus frappant que chez Apis mellifica car l'augmentation de poids des jeunes colonies correspond au même moment à une diminution générale des réserves pour les colonies âgées.

\section{VIII. - DISCUSSION ET CONCLUSION}

Une comparaison avec les travaux antérieurs d'auteurs brésiliens cités dans notre introduction va nous permettre 1 ) de dégager les apports originaux de ce travail et 2 ) de suggérer quelques réflexions sur certains résultats particuliers.

\section{Les essaimages}

Nogueira Neto eut le grand mérite de faire le départ entre les caractéristiques de l'essaimage d'Apis mellifica et ceux des Mélipones ou des Trigones, 
mais l'optique de son travail ne l'a pas conduit à analyser finement la dynamique de la population fille au cours de l'essaimage. Terada, de son côté, après avoir décrit les phénomènes observés dans la ruche mère au moment de la fièvre d'essaimage et l'activité de la nouvelle colonie pendant un mois, s'est contenté d'indiquer la composition de l'essaim définitivement installé dans son habitation : ses chiffres correspondent d'ailleurs assez bien à ceux que nous avons trouvé pour les Hypotrigones. Il a dénombré 704 jeunes abeilles, 283 vieilles ouvrières et 96 intermédiaires.

Notre travail, réparti sur plusieurs années d'observation a l'avantage de montrer la relativité de certaines constatations concernant le temps nécessaire à la colonisation d'un nouvel habitacle : ce temps peut être de 2 à 3 jours ou de quelques semaines suivant les colonies ou la période de l'année. Nous avons pu mettre en évidence les nombreux échecs d'essaimages même lorsque ceux-ci ont déjà atteint la troisième étape. Enfin, ce travail, tout en confirmant les observations de Nogueira Neto, montre les variations extraordinaires des populations des ruches au cours des différentes étapes des essaimages.

\section{Les étapes des essaimages}

Nogueira Neto, au Brésil, dans l'État de Saint Paul, signale des essaimages pendant toute l'année sauf en juin. Il est vrai que les périodes d'essaimage varient avec les espèces. Trigona $(N)$ testaceicornis essaime de janvier à octobre, semble-t-il, Trigona (Te) jaty d'août à janvier, etc. En résumé l'auteur pense que les essaims, qui doivent s'envoler durant toute l'année dans les parties chaudes de son pays, se font de plus en plus rares et même deviennent inexistants en hiver dans le sud.

Fonseca qui a étudié Trigona (Par.) subunda dans le même État, écrit que cette espèce doit essaimer toute l'année sur la pression des surpopulations et de la chaleur.

Les résultats que nous avons obtenus au Gabon semblent ne pas s'accorder avec les conclusions des deux auteurs précédents. En effet, le phénomène de l'essaimage, du moins en Afrique, est beaucoup plus complexe : il est lié, sans aucun doute, directement à la chaleur, mais aussi indirectement aux pluies, par l'intermédiaire des fleurs. Nous espérons mettre davantage ce fait en lumière lors d'une prochaine étude comparative des périodes d'essaimages au Gabon et en Côte d'Ivoire : si dans le premier pays ils n'ont lieu que pendant une partie de l'année, en revanche, dans le second ils doivent se produire en tous temps.

L'essaimage chez Apis mellifica et chez les Hypotrigones

Nous n'avons pas ici l'intention de reprendre et de répéter ce qu'ont écrit Nogueira Neto et d'autres auteurs après lui, mais nous voulons mettre 
l'accent sur un phénomène suggéré par la lecture du travail de Combs. Ce dernier auteur pesa des ouvrières d'Apis et mesura la concentration en sucre dans leur estomac. L'expérience répétée sur plusieurs dizaines de colonies venant juste d'essaimer montra que la charge des ouvrières était quatre fois plus grande que celle des ouvrières des colonies normales, c'est-à-dire n'essaimant pas. Cette surcharge des ouvrières essaimantes se manifestait d'ailleurs graduellement durant les 10 jours qui précédaient l'essaimage.

Ce phénomène est intéressant et il faut le rapprocher de ce que nous avons vu chez les Trigones. En effet, les Trigones de la nouvelle société restent en relation constante avec la colonie mère et rapportent régulièrement dans la jeune ruche tout ce qui est nécessaire à son bon départ. Mais, ici, les emprunts sont beaucoup plus variés que chez Apis puisqu'elles ramènent dans le nouveau nid à la fois de la cire, du miel et du pollen. Cependant le phénomène est semblable dans son essence, les nouvelles colonies d'Apis et celles des Trigones prenant chacune sur la colonie mère leur quote part de réserve. Mais ces deux groupes d'abeilles ont résolu le problème de manière différente.

Reģu pour publication en septembre 1976.

Eingegangen im September 1976.

\section{ZUSAMMENFASSUNG}

Trotz der Beobachtungen vieler Wissenschaftler des 19. Jahrhunderts dauerte es bis zu den Arbeiten von Nogueiro-Neto (1947, 1948, 1954) und von KerR (1951), bis die besonderen und wesentlichen Merkmale des Schwärmens bei Trigonen und Meliponen Gestalt annahmen.

Die vorliegende Arbeit ist das Ergebnis der Beobachtungen von zwölf Jahren. Wir haben uns bemüht, bestimmte, das Schwärmen von Hypotrigona betreffende Einzelheiten genauer zu bestimmen. Dazu benutzten wir einen Bienenstand mit mindestens hundert Völkern. Danach stellten wir die Schwarmzeiten in den äquatorialen Wäldern im Nordosten von Gabur. fest. Und schliesslich wird das Legeverhalten der Hypotrigona-Königin genau beschrieben.

Die Beobachtungen wurden mit Hilfe durchsichtiger Bienenkästchen aller Art (Glasröhren, Plexiglaskästchen usw.) durchgeführt. Die Beschaffenheit dieser Bienenkästen machte es möglich, eine Methode zur Überführung der Völker aus den hohlen Bäumen, in denen sie normalerweise leben, zu entwickeln.

\section{Die Schwarmzeit}

Durch regelmässiges Aufstellen der leeren Kästchen auf der Bienenbank unseres Bienenstandes während des ganzen Jahres war es möglich, die Schwarmzeit von Hypotrigona festzustellen. Die Versuchskästchen können von Mitte November bis Anfang Mai besiedelt werden. Das ausgesprochene Schwarmfieber stellt sich indessen im Dezember und Januar ein.

In Gabun ist demnach das Phänomen Schwarm bei Hypotrigona am Ende der grossen Regenzeit, der kurzen Trockenperiode und der kurzen Regenzeit in vollem Gange. Zu dieser Zeit haben die seit mehreren Monaten in ihren Kästen lebenden Völker ihre grossen Honig - und Pollenvorräte angelegt, und im Wald blüht es reichlich. Die Apis mellifica - Völker haben übrigens den gleichen Rhythmus wie die Trigonen, und die Einwohner von Gabun 
wissen, dass dies die beste Zeit ist, um den wilden Bienenvölkern die übervollen Honigwaben zu nehmen.

An dieser Stelle muss darauf hingewiesen werden, dass der Schwarmzyklus von Hypotrigona, selbst wenn man die jahreszeitlichen Unterschiede der verschiedenen Breitengrade berücksichtigt, nicht auf ganz Westafrika zu übertragen ist. Dies wird eine weitere Arbeit über das Schwärmen an der Elfenbeinküste zeigen.

\section{Die Abschnitte des Schwärmens -- Die Dynamik des Volkes}

Es war leicht festzustellen, dass das Schwärmen der Hypotrigonen in vier Etappen vor sich geht, in deren Verlauf man immer wieder einer mehr oder minder plötzlichen Veränderung in den Beziehungen zwischen der drei Altersklassen der Arbeiterinnen begegnet. Das relative Alter der erwachsenen Bienen lässt sich tatsächlich an der Farbe und an der Länge des Abdomens erkennen.

In der ersten Stufe des Schwärmens kommen alte Sammelbienen im neuen Nest an, die damit beginnen, die Wohnung vollständig einzurichten. Darauf fangen sie in Begleitung einiger jüngerer Bienen mit dem Bau von Vorratzellen an. Nun beobachtet man eine zahlenmässige Zunahme junger Arbeiterinnen und eine korrelative Abnahme der ältesten Bienen. Die bereits begonnenen Arbeiten werden fortgesetzt. Schliesslich beginnt die vierte Phase des Schwärmens mit der Ankunft einer unbegatteten Königin, die von sehr vielen jungen Arbeiterinnen begleitet wird. Zu diesem Zeitpunkt beginnt der Bau der Brutzellen. Die Königin wird begattet und beginnt mit der Eiablage.

\section{Das Legeverhalten der Königin}

Das Legeverhalten der Königin konnte in seinen Einzelheiten verfolgt und mit dem einer anderen afrikanischen Trigone, Trigona (Apotrigona) nebulata und mit dem zweier Arten der Neuen Welt verglichen werden.

Während die Königin sich ruhig verhält und von einem kleinen Hofstaat - etwa einem Dutzend Arbeiterinnen - umgeben ist, bauen Gruppen sehr junger Bienen gleichzeitig mehrere Zellen. Plötzlich unterbricht die Königin ihre Untätigkeit und läuft hin und her, hauptsächlich in Richtung Brut. Sie inspiziert häufig das Innere der im Bau befindlichen Zellen, wobei sie die Arbeiterinnen notfalls auseinandertreibt. So können bis zu zwei Stunden zwischen dem ersten Ortswechsel und der Ablage des ersten Eies vergehen. Diese Zeitspanne ist aufgeteilt in Ruheperioden der Königin und Umherwandern auf der Brut. Währenddessen fahren die Arbeiterinnen fort, an den Brutzellen zu bauen. Wenn diese ihre endgültige Form erlangt haben, wackelt die Königin lebhaft hin und her, schlägt rasch mit den Flügeln, schubst die Arbeiterinnen, die ihr im Wege sind oder auf den neuen Zellen warten, weg. Endlich hält sie auf einer neu gebauten Zelle an. Junge Arbeiterinnen entleeren dann rasch ihren Honigmageninhalt in die Zelle. Die Königin lässt ihre Antennen mit denen der Arbeiterinnen spielen, besichtigt das Zelleninnere, verlässt die Zelle ab und zu, kehrt aber stets sehr schnell zurück. Wenn das von den Arbeiterinnen abgesonderte, flüssige Futter die erforderliche Höhe in der Zelle erreicht hat, jagt die Königin die Arbeiterinnen davon und legt ein Ei in die Zelle. Die Zeit, die zum Füllen der Zelle gebraucht wird, die Anzahl der Arbeiterinnen, die sich über die Zelle beugen und die Zeitspanne zwischen zwei Eiablagen sind sehr verschieden. Gleich nachdem das Ei abgelegt ist, fängt eine einzige Arbeiterin aus dem Kreis derer, die sich auf den Brutzellen befinden, damit an, die Zellen zu verdeckeln. Niemals wurden Arbeiterinnen beim Eierlegen beobachtet, und ebensowenig konnten Königinnen oder Arbeiterinnen beim Eierfressen beobachtet werden, obwohl bei einigen Arbeiterinnen ein Ei in den Ovariolen gefunden wurde. Es ist indessen bekannt, dass bei verschiedenen Meliponen und Trigonen häufig über solche Beobachtungen berichtet wurde. 


\section{Die äussere Entwicklung des neuen Volkes}

In Makokou haben wir etwa vier Monate lang regelmässig die Völker vom Vorjahr und die neu aus Schwärmen entstandenen in ihren Beuten gewogen. Dabei wurde festgestellt, dass das Gewicht der jungen Völker regelmässig zunimmt, und dass während der gleichen Zeit die alten Völker an Gewicht verlieren. Dieser Gewichtsverlust der alten Völker ist während der gesamten Schwarmperiode festzustellen. Er hält auch in den folgenden Monaten noch an.

Am Schluss dieser Arbeit werden die jetzigen Erkenntnisse über das Schwärmen der Trigonen mit den Beobachtungen verglichen, die Cомвs neuerdings bei Apis mellifica gemacht hat. Beide Bienengruppen haben das Problem der Futterversorgung ihrer neu entstehenden Völker auf unterschiedliche Weise gelöst. Beide entnehmen dem Muttervolk ihren Anteil an Futtervorräten, d.h. bei Apis mellifica Honig, bei Trigona Wachs und Pollen. Aber während das neue Trigona - Volk lange Zeit in enger Verbindung mit dem Muttervolk bleibt, um. sich mit Vorräten zu versorgen, nimmt Apis mellifica die Vorräte auf einmal mit und zwar im Honigmagen der Abschwärmenden, die sich zur Abreise ohne Wiederkehr aus der Beute ins Freie stürzen.

\section{RÉFÉRENCES BIBLIOGRAPHIQUES}

Bassindale B., 1955, The Biology of the Stingless Bee, Trigona (Hypotrigona) gribodoi Mag. Proc. Zool. Soc. London, 125, (1), 49-62.

Camillo C., 1972, Alguns aspectos de comportamento de Melipona rufiventris rufiventris Lepeletier. Hom. a W. E. Kerr, Sao Paulo, Brésil; 57-62.

Charles-Dominique P., 1971, Éco-Éthologie des Prosimiens du Gabon. Biol. Gab., 7, (2), 121-228.

Сомвs G. F., 1972, The engorgement of swarming workers honeybees. J. Apic. Res., 11, (3), 121-128.

Darchen R., 1972, Écologie de quelques Trigones (Hypotrigona sp) de la savane de Lamto (Côte d'Ivoire). Apidologie, 3, 341-367.

Fonseca L. I., 1975, On swarming activity in Meliponina. Résumé d'une communication au 25e Congrès d'Apiculture d'Apimondia, Grenoble, France.

Sakagami S. F., Zucchi R., 1974, Oviposition behavior of two dwarf Stingless Bees, Hypotrigona (Leurotrigona) muelleri and Hypotrigona (Trigonisca) duckei, with notes on the temporal articulation of oviposition process in Stingless Bees. J. Fac. Sc., Hokkaido Univ., 19, (2), 361-421.

Schwartz H. F., 1948, Stingless Bees (Meliponidae) of the Western Hemisphere. Bull. Mus. Natur. Hist., New York, 90, 546 p.

Sмгтн F., 1968, Observations on the economy of Brasilian insects, chiefly Hymenoptera, from the notes of M. Peckolt. Trans. Ent. Soc. London, 15, 323-327.

Spinola M., 1840, Observation sur les apiaires méliponites, Ann. Sci. Nat., 2, (13), 116-140.

TeradA Y., 1972, Enxameagem em Friesemelitta varia Lepeletier (Hymenoptèra, Apidae). Hom. W. E. Kerr, Sao Paulo, Brésil; 293-300.

Kerr W. E., 1951, Bases para o estudo da genetica de populaçoes dos Hymenoptera em general e dos Apinae sociais em particular. An. Esc. Sup. Agric. Luis de Queiroz, 8, 219-354.

Moure J. S., Nogueiro-Neto P., Kerr W. E., 1958, Evolutionary problems among Meliponinae (Hymenoptera, Apidae). Proc. X Intern. Congr. Entomol., 2, 481-493.

Noguerra-Neto P., 1951, Stingless Bees and their study. Bee World, 32, (10), 73-76.

Nogueira-Neto P., 1954, Notas bionomicas sobre Meliponeos : III Sôbre a enxameagem. Arquiv. Mus. Nac., Rio de Janeiro, 42, 419-451.

Nogueira-Neto P., 1970, A criaçâo de Abelhas indigenas sem ferrâo. Tecnapis, Sao Paulo, Brésil; 365 p.

Sakagami S. F., 1971, Ethosoziologischer Vergleich zwischen Honigbienen und stachellosen Bienen. Z. Tierpsychol., 28, 337-350. 